\title{
The Emergence of Intelligence in the Universe
}

\author{
SIR FRED HOYLE
}

\author{
The question is - On what fraction \\ of planets in how many millions of cases can we expect \\ not just life, but intelligence, to emerge?
}

There may be forms of life of which we are totally unaware and of which we can barely conceive. For example, you might speculate on the possibility of life being based on the properties of nuclear matter, life in exceedingly dense space-several hundred million tons of it in a volume the size of a sugar cube. But at our present level of understanding, this would be more a matter for a science fiction story. So let me bypass all the strange things that might possibly exist in the universe and discuss the emergence of life and intelligence in terms of chemical life based on the properties of the carbon atom.

In contrast to the situation only a few decades ago, scientists today believe that they know quite a bit about this kind of life, which is based on complex chemical reactions, with substances called proteins playing a dominant and important role. You know that in ordinary dietary terms we are supposed to have a daily intake of proteins in our food. It is, however, important to realize that we do this not for the sake of the particular proteins that we eat but for the basic substances of which these proteins are made.

What happens is that the proteins we eat are first broken into their constituents, known as amino acids, and these amino acids are then built - within our own bodies-into the proteins required specifically by our kind of creature -the human being. The dog, which operates similarly, uses the same basic amino acids, building them into protein structures peculiar to itself. And so for all living animals. We all use the same basic amino acids, but we arrange them individually according to our own separate needs.

How does each animal build just what is right for itself? Nowadays, biologists even understand the answer to this crucial question. Each of us contains within himself a kind of vast chemical blueprint that is simply copied time and time again as our kinds of proteins are made, in order to serve our separate bodily functions.

But it's not my purpose to develop the chemical basis of life in any detail. I merely want to emphasize that life is now seen to be based on a complex but well-ordered form of chemistry, which is to say, on the relation between various kinds of atoms. The relations between atoms are described by the science of physics-by methods that are well understood. Indeed, no very deep knowledge of physics is required in order to calculate how an atom of sodium and one of chlorine bind themselves to form a molecule of sodium chloride, or common salt.

The relevant basis for this kind of understanding was discovered 50 years ago in the work of Heisenberg and Schrödinger, and that of Wolfgang Pauli. It came at the beginning of that revolution of physical thinking known as quantum mechanics. Yet, although we believe we understand the basic principles on which molecules are constructed from atoms, it's beyond our ability to calculate the details of any but the simplest molecules. It would be possible to calculate the properties of a very simple molecule like sodium chloride with reasonable precision, but it would be quite beyond our powers to calculate the detailed properties of a protein containing a thousand or more amino acids.

Now all this is very odd and very interesting. By restricting ourselves to the study of simple systems we seem to be able to discover rules according to which the world is constructed on a much more complex scale. Could it be 
that our inability to follow through in understanding this more complex scale is a temporary handicap-one that will eventually be swept aside as science advances? I doubt that this will turn out to be so. I doubt that there is any simpler description of the universe than the universe itself.

The usual concept of the scientist that eventually he'll be able to build a simple model of the universe that will serve to describe with accuracy the behavior of the actual

\section{People who'd never dream of strangling a dog \\ don't hesitate to swat a mosquito}

universe is, I believe, a chimera. What we can do, however, is build models that give a satisfactory description of limited aspects of the universe. It's when we come to demand full detail that the trouble arises. We can manage to deal with a molecule of sodium chloride perfectly well, and in doing so we gain insight into the general properties of proteins, and of even larger biochemical structures than the proteins. But we fail in our endeavor to describe detail. In short, our brains, our understanding, permit us a perceptive view of the universe but not a complete view-nor, I believe, will they ever permit us a complete view.

It is subject to this inherent limitation that I want to consider what can be said, firstly, about the emergence of life in the universe, and secondly, about the emergence of intelligence. And then, by combining what we find about these two topics, I want to give some thought to what the outcome for life here on Earth may turn out to be in the centuries and millennia that lie ahead of us.

Although I described amino acids as being much less complex in their structure than proteins, and although the proteins themselves are much less complex than the remarkable long-chain molecules that carry our genetic heritage, it's important to realize that even amino acids are complex compared with the substances that life evolved from in the first place. These were molecules such as water, hydrogen cyanide, carbon dioxide, and possibly ammonia; in fact, just the kind of molecules that astronomers have discovered in vast numbers within the gas clouds of the Milky Way.

When we look at galaxies other than our own, we can see evidence of clouds of gas and dust. We have no reason to believe that other galaxies are any different from ours, and when we come inside our own galaxy, we can look at some of the detail of clouds and gas that contain the molecules out of which we believe life was born.

In view of this widespread diffusion of the basic lifeforming molecules everywhere in the galaxy - and in other galaxies, as we believe - one would naturally suppose that life is likely to be widespread throughout the universe. The basic physical laws that permit the chemistry of life are the same in other places. Similar structures to ourselves can therefore be expected simply because of the vast profusion of planets and stars.

There are rather more than 100,000 million stars in our galaxy alone. A large fraction of them possess the characteristics that astronomers believe to be associated with the occurrence of planetary systems. In other words, there are strong reasons for thinking that a considerable fraction of the stars in our galaxy-perhaps 50 percent of thempossess planets moving around them. So, in considering the emergence of life on the galactic scale, we have to think of something of the order of 100,000 million planetary sites. Life might also arise in ways other than on planetary surfaces, but let's just keep to the kinds of things we know something about.

Not all planetary systems will be suitable for the emergence of life. Among the planets of our own solar system, only our Earth is likely to possess life. Maybe there are people who would like to challenge this, but when you look at the Earth, you can see why it's possible to have some skepticism as to whether life is likely to exist on the other planets. The Earth is completely differentenormous atmospheric movements and a very different kind of color. The Earth is so manifestly different from the others as to bring home to us the fact that, had the Earth not been present in our system-had the Sun possessed eight planets, instead of nine, without the Earth -then the solar system would, I rather imagine, be sterile.

Among other systems of planets we must suppose that there will be some that will be sterile. What fraction is this likely to be? In one sense the answer to this question is quite uncertain, but in another sense our uncertainty is probably irrelevant to a more important question: Having allowed for all the astronomically and chemically unfavorable cases, do a large number of suitable sites for the emergence of life still remain?

After all, we had 100,000 million possibilities to start with. If only 10 percent of these are astronomically suitable, and if only a further 10 percent possess an appropriate kind of chemistry, as many as 1,000 million favorable sites still remain. It seems, then, rather unlikely that the favored fraction would be less than this - 1,000 million favored sites for the origin of life.

The first step toward understanding the origin of life is reasonably well understood. This is the step in which the 
simple molecules that occur in huge quantities in our galaxy and other galaxies are built into more complex molecules-substances containing a moderate number of atoms (say 30 to 100 atoms) like the amino acids. The essential feature of this first step is that it supplies a store of energy that can then be used to drive more complex systems. The source of the energy must be the light that shines on the planet from the primary central star.

This first step doesn't seem difficult to achieve, and most chemists and biologists seem to have little doubt that it would take place in nearly all cases. So far, so good. Yet, with such an energy store we're still far from the synthesis of the exceedingly complex molecules on which life itself is based. Much work is going on today, seeking to discover how the first biological cell—-the first cell able to reproduce itself--came into being.

Until more is known about this second step, it's still too early to make a quantitative estimate of the probability of life emerging in a particular place. There could be barriers requiring highly improbable circumstances that could eat into our 1,000 million cases to a substantial degree. But on the other hand, you can say that it's been the experience so far that estimates of probabilities seem to rise-not to fall-as more becomes known about the problems involved. This has certainly been the case on the astronomical side. The evidence regarding the molecular chemistry of the interstellar clouds shows the same thing on the chemical side, and a reasonable guess is that a similar situation will arise as more becomes known about the biological details.

Given the first living cell, much still remains before life as we know it is forthcoming - particularly before intelligent life can emerge. Even on the Earth, complex life forms, creatures aggregated from very many cells, were a long time in coming. It's only about 500 million years ago that you begin to get the complex life forms that we normally think of as living creatures.

This distribution of life with respect to time on the Earth seems to indicate that until comparatively recently a barrier of some kind existed - a barrier that prevented more complex forms of life than single-cell bacteria and blue-green algae from existing. We can speculate what this barrier might have been, and I find it rather impressive that there is a temperature correlation involved in the very early life forms. Strikingly, they were all forms that survived under high-temperature conditions-bacteria right up to the boiling point of water, the blue-green algae to about $75^{\circ} \mathrm{C}$, the various kinds of fungi to about $60^{\circ} \mathrm{C}$ - suggesting that the Earth in its early history may have been too hot to permit the existence of any but singlecelled creatures with highly protective cell walls. If this is true, it has far-reaching astronomical implications.

But let's move on to the emergence of intelligent creatures, which is what we are really interested in. The question is, on what fraction of planets in how many of our millions of cases can we expect, not just life, but intelligence to emerge? Indeed, our emotional attitude to life isn't really a chemical matter at all. Although the difference between a well-loved person being alive and being dead may depend on certain subtle chemical processes, this isn't at all the way we feel about it. Most people who'd never dream of strangling a dog don't hesitate to swat a mosquito. Yet the chemistry of the mosquito is basically the same as that of the dog.

The situation is that we distinguish between "higher" and "lower" animals according to the complexities of the nervous systems with which animals are endowed. A nervous system is basically electrical in its operation, with an animal made up of a chemical system together with an electronic one. The more the electronic part of this summation dominates, the higher we judge the animal to be in the zoological evolutionary scale. And the more the electronic system happens to match our own system, the better the animal.

Among humans, the more similar the other person's electronic system is to our own the better regarded, or the better loved, the person is. So you can see that similarity, or otherwise, in the electronic part distinguishes the category of "us" from the category of "them." Further-

\section{The logic of evolution forces development of the most deadly weapon of all - a thinking brain}

more, at a certain level of electronic complexity, we rather arbitrarily introduce the notion of "intelligence"-a level set just a little below our own capacity. So, essentially, as a matter of definition, any creature with an electronic system more complex than our own would be endowed with high "intelligence."

Animals aren't regularly able to synthesize the amino acids and sugars that are essential to them, as plants do. Animals must therefore acquire these substances either by eating plants or by eating other animals. Basically, all animals are scroungers, living on the stored chemical potentialities that others have first accumulated. It was precisely to assist in the process of scrounging that the electronic systems possessed by.animals developed. And since the better the electronic system the better the scrounger, biological evolution has operated steadily, over millions of years, to increase the level of complexity 
of animal electronics. And since we judge the level of an animal by the complexity of its electronics, it follows that the higher the animal the greater the scrounger-with man himself sitting at the top of the pyramid.

The electronic system in man has indeed become so subtle that our scrounging for energy, in particular, has now extended well beyond the eating of plants and other animals. We scrounge extensively today on nonliving materials. The discovery of fire made use of the decay products of trees in the form of wood. The burning of coal and oil were further steps along the same path. Now, in the modern nuclear plant, we've attained to the use of entirely nonorganic materials as an energy source. This access to nonanimal sources of energy has developed with increasing rapidity to a point in our modern society where we can clearly see that either some more restrained pattern of behavior must be applied in future years, or the evolution of our species will end itself in a catastrophic social explosion.

It's in these evidently crucial circumstances that we've begun to wonder how things may have fared with other creatures on other planets moving around other stars. We've even begun to wonder about the possibility of communicating with them.

Interstellar communication, as we call it, raises many questions-some technical, some of quite general interest. As far as can be seen at the moment, the only feasible mode of communication between creatures living on different planets moving around different stars would seem to be by a radio link.

\section{We are living today not on the brink of social disaster, but actually within the disaster itself}

A vast array of 900 individual radio telescopes, each with a diameter of 100 meters, has been proposed. The idea is that such an array would give the best expression to our present ability to communicate on an interstellar scale. This proposal has been aptly named Project Cyclops ("Hello Out There," E\&S-March-April 1973; "The Search for Extraterrestrial Intelligence" by Bernard M. Oliver, $E \& S-D e c e m b e r$ 1974-January 1975). It is worth noticing that actual physical travel by men in space, while it may just be possible, doesn't seem really very good. Even if it were possible, physical travel would take much more time than an interchange of messages through a system like Project Cyclops.
The difficulties of physical travel to distant stars might seem at first sight to be a decline in romantic possibility, a loss of richness in the scheme of things. But a little thought shows that precisely the opposite is true. If physical travel from one planetary system to another were feasible, then the first creatures to become technologically capable of space travel would be likely to spread themselves throughout the galaxy-just as science fiction writers are always imagining our human species to do. It would be only too likely that the galaxy would come in that way to have only one form of intelligent creature, and this indeed would be a loss of richness.

But if you take the view that space travel is not possible, creatures in one planetary system can't interfere with the physical development of creatures in another system. Many possibilities, with much potential richness, are then permitted.

Are other intelligent creatures really likely to exist? From what I've already said there seems to be little if anything in our own solar system that is due to distant chance. To be sure, if we knew there to be only one other planetary system in our galaxy, the odds would be against it containing a planet like the Earth, at an appropriate distance from an appropriate central star, with a similar rotation speed, a similar axial tilt, similar chemistry, and so on. But the chance of a similar situation isn't all that small-perhaps one in ten or one in a hundred, but probably not much less than that. And since we haven't just one planetary system to consider, but some 100,000 million of them, we have no real difficulty, I think, on this score.

But would life in such places become intelligent in the sense that I've been describing intelligence? Well, from our rather brief consideration of the nature of an "animal," it seems that the development of an electronic system would very likely occur for all animals everywhere. In the need to search for food, eyes would be a normal development. Animals with eyes are likely to prey upon each other, since the logic of evolution forces the development of weapon systems, claws, teeth, and ultimately a thinking brain - the most deadly weapon of all. The logical sequence leading to the emergence of a thinking brain appears to me inevitable, and I think we can expect this to have happened quite generally.

I come now to what appears to be the most uncertain question of all. Given a suitable planet, given the origin of life, given the emergence of intelligence to a level at least equal to our own, for how long on the average can one expect such an intelligence to persist? Even if intelligence arises in as many as a million cases in our galaxy, there will still be very few such cases around at the present moment unless high intelligence, once it arises, persists for more than 10,000 years. This is simply because the age of our galaxy, the time span over which intelligence 
emerges, is very long indeed-about 10,000 million years. So, unless intelligence lasts once it arises, there will be very little overlap in time between its brief emergence on one planet and its emergence on another planet.

The thought that our capacity to execute a project of the technological quality of Project Cyclops might only last for 10,000 years seems, to begin with, to be a rather pessimistic assessment of the future of the human species. But in view of the state of our present-day society, is it not a rather optimistic assessment? When you contemplate the huge human populations that have grown with startling suddenness in only a century, when you contemplate the excessive modern pressure on natural resources, I think it's hard to put much confidence in a future extending more than a few decades. Devastating crises, one feels, must surely overtake the human species in no more than another hundred years. We're living today, not on the brink of social disaster, as we often tend to think, but actually within the disaster itself.

We've seen that the phenomenon of "intelligence" is the outcome of aggressive competition. Intelligence and aggressiveness are coupled together inevitably by their biological association. An intelligent animal anywhere in the galaxy must necessarily be an aggressive animal, and must eventually be faced at some time by the same kind of social situation as that which now confronts the human species. Inevitably, then, "intelligence" contains within itself the seeds of its own destruction. This leads to a very critical question: Can any solution be found for this inherent difficulty?

We find it hard to believe that our civilization could ever come to a final end, with no further rise of humanity into the future. A belief in the extended future of our species is almost a religious faith, which I think we all possess. Certainly it was so with me in the past. Although my reason told me that all was far from well, I continued to believe that somehow it would all come out right in the end. Then one day I was suddenly struck with an ominous thought. We all know that nature is exceedingly prolific. There are many millions of galaxies, many millions of stars, perhaps many millions of intelligent kinds of creatures. What nature does not do is to demand success of every trial, every attempt. In fact, it is just the opposite. One could say that nature proceeds by many trials, just because in so few cases is there success. In a manner of speaking, perhaps this is the reason for there being so many planets-so many attempts at an intelligent creature -because, among intelligent creatures, very few of them are ever going to make it.

So, far from us muddling through the future in some way, perhaps the chance of our coming through at all is very small. A nasty, unpleasant thing, you might say, to be putting forward. Well, if what I've just been saying is wrong, it doesn't matter at all. Life will roll on, and events will show that this is a lot of nonsense. But if what I've just said is right, then the very worst thing for us to do is to ignore the dangers ahead. If the danger is really there with high probability, then the most essential step in reducing the probability is to be very clearly aware of the danger. The very worst thing would be to go on without heed of it. So I'm not making apology now for drawing your attention to these issues.

Let me end what I have to say by discussing very briefly how we suppose things will have to go here on the Earth if the human species is to be one of the rare creatures that makes it to some higher, some more stable level of intelligence. Very clearly, a much lower population level will be needed, pressing only gently, if at all, on the resources of the Earth. To achieve such a situation will be a crisis that faces us over the coming decades and centuries. I have now come to believe that this will be a crisis every bit as critical as any of those that have occurred over our long past history. Only after the resolution of this crisis can we look forward to a time span for our species even as long as 10,000 years. Perhaps then we can think in terms of a very much more extended futuremillions rather than thousands of years.

So let's turn back to the matter of interstellar communication. For creatures with millions, not thousands, of years ahead of them, a necessary interval of several hundred years between the transmission of a message and the reception of a reply to it wouldn't be a serious impediment. There would be ample time for many messages to be interchanged. No species in this situation would, I think, hesitate, as we do now, to search our galaxy for the other intelligences which must surely have emerged and which may have climbed over the difficulties that confront our human species today. 